\title{
MaMIMO User Grouping Strategies: How much does it matter?
}

\author{
Andrea P. Guevara, Sibren De Bast, Sofie Pollin \\ Department of Electrical Engineering, KU Leuven, Belgium \\ Email:\{aguevara,sdebast,spollin\}@esat.kuleuven.be
}

\begin{abstract}
In a Massive MIMO communication system, a large number of antennas increases the spectral efficiency; however, if this number is fixed, then the number and selection of users influence the performance of the system. In this work, an ultra-dense massive MIMO channel database is obtained for different antenna geometries, centralised and distributed scenarios, and propagation conditions. The greedy search selection of multiple users to form a group is analysed under capacity and simplified heuristic approximations. Experimental results show that the grouping strategy has a significant impact on the nLoS scenario only when a small number of users is served (multiplexing regime). However, in LoS cases, the distribution of the base station antennas has a higher impact on the system performance than the selection of the users.
\end{abstract}

\section{INTRODUCTION}

One of the key technologies for future wireless communication networks is massive MIMO. In such systems, a large number of antennas $M$ is deployed in a base station, serving coherently multiple users $K$ at the same time and frequency. This allows to achieve impressive spectral efficiency (SE) records not only in theory [1], but also experimentally [2].

In a massive MIMO system, the cell spectral efficiency increases along with the number of users. This increase is steady when there is a small number of $K$ users to be served, due to the multiplexing gain, also known as multiplexing regime. However, if $K$ grows, the inter-user-interference (IUI) and pilot overhead impact the sum SE until it reaches a saturation point, this period is called the saturation regime. Afterwards, during the scheduling regime, any additional user does not contribute to the sum SE [3].

One solution to serve more users is to increase the number of antennas at the base station. Theoretically, in a massive MIMO system, the number of antennas can increase infinitely; however, in real scenarios $M$ is a constraint due to hardware complexity, cost and power consumption [4]. The relation between the number of antennas and users $M / K$ has been studied thoroughly in simulated scenarios in [5], [6]. The authors conclude that there is not a fixed optimal ratio, as this relation depends on the channel estimation process, pilot overhead and the combining vector or beamforming schemes.

In [7], the impact of the distribution of antennas on user scheduling is studied as an additional variable. The authors show an increment of the sum SE when the antennas are homogeneously distributed as two URAs (Uniform Rectangular Array). To the best of our knowledge, there is not an experimental study that considers different antenna array geometries, its distribution and the impact that it causes during user scheduling.

\section{A. Contribution}

In this work, a massive MIMO single-cell experiment is carried out, under different antenna array geometries and distributions, for two scenarios with and without Line-ofSight (LoS). The collection of the channel data is carried out for 120 users; thus, the base station must choose a subset of users from this pool using different greedy search user grouping techniques. The greedy search follows local performance criteria, where one is the optimal capacity metric, and two heuristic techniques based on channel gain and user distance. Given the fact that our base station has 64 antennas, we assume that a maximum of 64 users can be served simultaneously. The spectral efficiency and user fairness are the metrics used to analyse the performance.

The following notations are used throughout this paper: $\mathbf{x} \in \mathbb{C}^{\mathrm{M}}$ is a $M \times 1$ complex vector, $\mathbf{X} \in \mathbb{C}^{\mathrm{M} \times \mathrm{N}}$ is an $M \times N$ complex matrix. $\mathbf{x}^{\mathrm{H}}$ and $\mathbf{x}^{\mathrm{T}}$ are a transpose and conjugatetranspose of vector $\mathbf{x} .\|\mathbf{x}\|$ is the Euclidean norm of $\mathbf{x}$.

\section{SYSTEM MODEL}

In the following section, a description of the system and the wireless channel is presented, followed by a brief explanation of the channel estimation procedure. To finalise, a definition of the Zero-Forcing (ZF) combining vector and the uplink spectral efficiency is detailed.

\section{A. System Model and Wireless Channel}

In this study, we consider a single-cell massive MIMO Time Division Duplex (TDD) system. This cell has a base station with $M$ antennas, which are deployed in a centralised or distributed manner, and $K$ single-antenna users each transmitting with power $p$.

The wireless channel vector between user $k$ and all the base station antennas is denoted by $\mathbf{h}_{k} \in \mathbb{C}^{\mathrm{M}}$. Therefore, the path gain per antenna is given by:

$$
\beta_{k}(m)=\mathbb{E}\left\{\left\|\mathbf{h}_{k}(m)\right\|^{2}\right\} .
$$

The individual channel correlation matrix for user $k$ is expressed as:

$$
\mathbf{R}_{k}=\mathbb{E}\left\{\mathbf{h}_{k} \mathbf{h}_{k}^{\mathrm{H}}\right\}
$$




\section{B. Channel Estimation}

The channel estimation process is carried out in every coherence time block, using an uplink training signal. This training signal contains individual sequences of orthogonal pilots $\left(\varphi_{p} \in \mathbb{C}^{\tau_{p}}\right)$ that are sent simultaneously by each user through its channel. Once this training signal reaches the base station, it enables the estimation of the channel for each user with their conjugate pilot. The estimated channel matrix $\hat{\mathbf{H}}$ contains the estimated channels of all $K$ users, $\hat{\mathbf{H}}=\left[\hat{\mathbf{h}}_{1} \ldots \hat{\mathbf{h}}_{k} \ldots \hat{\mathbf{h}}_{K}\right]$. Similar to (2), we can obtain the estimation error channel matrix as follows:

$$
\mathbf{C}_{k}=\mathbb{E}\left\{\left(\mathbf{h}_{k}-\tilde{\mathbf{h}}_{k}\right)\left(\mathbf{h}_{k}-\tilde{\mathbf{h}}_{k}\right)^{\mathrm{H}}\right\} .
$$

The main errors that can be contained in $\mathbf{C}_{k}$ are due to noise and pilot contamination; this occurs when pilot tones $\varphi_{p}$ are re-used by multiple users. In this paper, pilot contamination is not study, and only error due to noise will be considered.

\section{Uplink Transmission and ZF Combining Vector}

During uplink data transmission, all users send individual signals $(s)$ over their channel, which are coherently combined. Thus, the received uplink signal at the base station is:

$$
\mathbf{y}_{j}=\sqrt{p} \hat{\mathbf{h}}_{k} s_{k}+\sum_{\substack{k=1 \\ k \neq j}}^{K} \sqrt{p} \hat{\mathbf{h}}_{j} s_{j}+\mathbf{n}+\zeta .
$$

If we consider $s_{k}$ as the desired signal to be estimated in (4), one can relate the second term as the interference from all the other users. The third term in this equation is the noise $\mathbf{n} \sim \mathcal{C N}\left(\mathbf{0}, \sigma^{2} \mathbf{I}\right)$, where $\sigma$ is the noise variance. The last term is a variable that contains the channels estimation errors. To decorrelate the required signal from all the undesired ones in (4), the base station requires a combining vector.

In the proposed case study described in Section III-A, we assume a high Signal-to-Noise-Ratio (SNR) for all users. Accordingly, Zero-Forcing (ZF) is a suitable combining vector (v) [3]. Due to its orthogonality to the inter-cell interfering channels, ZF suppresses these undesired channels as follows:

$$
\left(\hat{\mathbf{H}}^{\mathrm{H}}\right) \mathbf{V}=\mathbf{I}_{K} \cdot
$$

Where $\mathbf{V}=\left[\mathbf{v}_{1} \ldots \mathbf{v}_{K}\right]$ is the $\mathrm{ZF}$ combining matrix. The identity matrix $\mathbf{I}_{K}$ contains only the desired signals of all users when there is perfect channel estimation, otherwise, $\mathbf{I}_{K}$ will contain additional interferencing signals. To achieve (5) the $\mathrm{ZF}$ combining matrix is given by:

$$
\mathbf{V}=\hat{\mathbf{H}}\left(\hat{\mathbf{H}}^{\mathrm{H}} \hat{\mathbf{H}}\right)^{-1} .
$$

ZF is only possible when the matrix $\mathbf{H}^{\mathrm{H}} \mathbf{H}$ has full rank, which only occurs when $K \leq M$.

\section{Spectral Efficiency}

To quantify the successfully received uplink data, we consider the uplink ergodic channel capacity of user $k$, that is lower bounded by $\mathrm{SE}_{k}$ :

$$
\mathrm{SE}_{k}=\tau \mathbb{E}\left\{\log _{2}\left(1+\gamma_{k}\right)\right\} \quad[\mathrm{bits} / \mathrm{s} / \mathrm{Hz}] .
$$

$\tau$ is the fraction of the frame used for uplink data, where the remainder of the frame is used for pilot information. And $\gamma_{k}$ is the signal-to-interference-plus-noise ratio (SINR) of $k$ user. If MMSE channel estimation is used, then:

$$
\gamma_{k}=\frac{p\left|\mathbf{v}_{k}^{\mathrm{H}} \hat{\mathbf{h}}_{k}\right|^{2}}{\sum_{\substack{j=1 \\ k \neq j}}^{\mathrm{K}} p\left|\mathbf{v}_{k}^{\mathrm{H}} \hat{\mathbf{h}}_{j}\right|^{2}+\mathbf{v}_{k}^{\mathrm{H}}\left(\sum_{j=1}^{\mathrm{K}} p \mathbf{C}_{j}+\sigma^{2} \mathbf{I}_{M}\right) \mathbf{v}_{k}} .
$$

Where $\mathbf{v}_{k}$ is the combining vector required to obtain the desired signal $s_{k}$ from (4).

\section{CASE Study ANd ExPerimental Scenario}

This section describes an indoor ultra-dense scenario that is used as a case study, followed by a massive MIMO experiment adjusted to the mentioned scenario.

\section{A. Case Study}

This work is an application to the proposed showcase 2: Low Latency Industrial Communication, as part of the "Orchestration and Reconfiguration Control Architecture" (ORCA) project [8]. This showcase describes a futuristic industrial scenario, where the transportation of multiples products could be automated, with the aid of robots.

A central cloud system will be able to make well-balanced traffic decisions based on information gathered by a large number of ambient sensors. Those stationary sensors could be deployed on the ground on strategic locations to monitor the presence of robots. The main goal of this closed-loop control application is to offer higher performance in real-time for indoor mobility.

\section{B. Massive MIMO Experiment}

A massive MIMO experiment is carried out to emulate an indoor industrial scenario where traffic sensors (or users) will be deployed on the ground in a grid distribution with a separation of 25 and $30 \mathrm{~cm}$. To create this scenario, we use four XY positioners which hold a dipole antenna each, one universal software radio peripherals (USRPs) controls a pair of antennas as independent users data streams. In Fig. 1, USRP1 controls the antennas placed on XY positioners 1 and 3; and USRP2 the dipole antennas of XY positioners 2 and 4 .

The positioners moved synchronously to 30 different positions every 30 seconds, with simultaneous and continuous signal transmission. The received uplink signal, generated by the USRPs, was collected and processed by a 64-antenna massive MIMO base station for each of the 120 positions, 


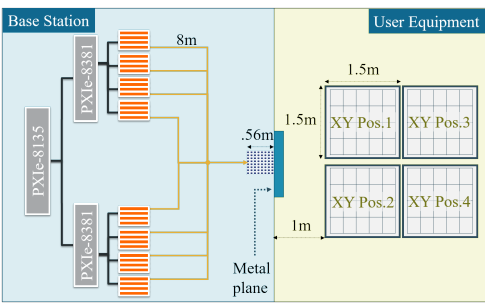

(a) Los and nLoS scenario deployment for a URA antenna array.

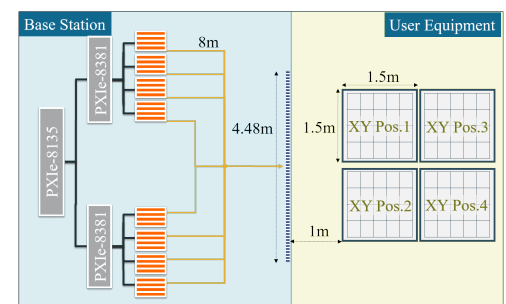

(b) LoS scenario deployment for a ULA antenna array.

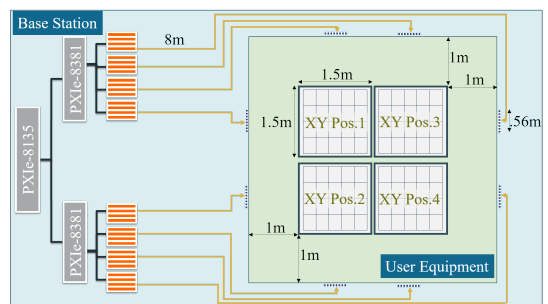

(c) LoS scenario deployment for 8 distributed ULA (D-ULA) antenna array

Fig. 1. Antenna deployment for the massive MIMO experiment.

then the channel for each position is saved to be process offline. During the experiment, the base station is controlled in real-time by the LabVIEW Communications MIMO Application Framework 1.1.

One of the main characteristics of the 64 patch antennas at the base station is their modularity. During this experiment, we were able to deploy three types of antenna topologies:

- Uniform Rectangular Array (URA): The 64 antennas were placed in an array of $8 \times 8$ antennas, Fig. 1(a). This centralised array was deployed at a one-meter distance of XY positioners 1 and 2, for the Light-of-Sight (LoS) scenario. While for the non-LoS (nLoS) scenario, a metal plane was deployed in front of the array.

- Uniform Linear Array (ULA): The 64 antennas were placed next to each other to form a $1 \times 64$ array of $4.48 \mathrm{~m}$. Fig. II-D. This array was deployed at a distance of 1 meter from XY positioners 1 and 2. Only the LoS scenario is consider for this topology.

- Distributed ULA (D-ULA): In this distributed scenario we deployed homogeneously 8 different arrays of $1 \times 8$ antennas around the outskirts of the $\mathrm{XY}$ postioners, as shown in Fig. II-D. The distance between each array and the closest side of the positioners is 1 meter. This topology is analysed for the LoS case.

The wireless data collection for the 120 locations was carried out for each of the antenna distribution mentioned above. It is worth mentioning that the same transmit power was set during all the experiments.

\section{USER GRouping Strategies}

Let $\mathcal{S} \triangleq\{1, \ldots, S\}$ represent the set of all the users from the previous experiment. To satisfy the necessary condition given by the ZF combining vector and to achieve a canonical Massive MIMO network $(M / K \geq 1)$ [3], we propose three greedy search user grouping strategies to select $K$ users out of the total $S$ users, as $x \triangleq\{1, \ldots, K\}, x \in \mathcal{S}$.

\section{A. Capacity-based User Grouping}

To compare the performance of the proposed ranking heuristics, we contrast the SE achieved when selecting the next user in the group using the capacity achieved when adding that user. While this method is not optimal, as it can still result in a local optimum, the capacity metric is the best greedy search metric when optimizing SE. Under the $\mathrm{ZF}$ combining vector, it is given as:

$$
\underset{k \in \varkappa}{\arg \max } \sum_{k}^{K} \mathrm{SE}_{k} .
$$

This greedy selection technique requires a huge computational complexity as capacity needs to be computed for all to be grouped users in each iteration. Below are shown empirical methods that do not require a demanding computational cost.

\section{B. Distance User Grouping}

Using this heuristic user grouping technique, the users will be ranked based on their distance to their closest antenna. The $K$ users with the smallest distance are then selected. It is worth mentioning that for the described experiment, it is feasible to obtain the exact location of the users with the aid of the XY positioners, however, to generalise this technique we need to apply a precise method to estimate the position of each user. Multiple techniques to localise users based on the estimated channel have been studied broadly. For example, in [9], the location of the users is calculated with very high accuracy and is applied to similar scenarios.

\section{Channel Gain User Grouping}

This sub-optimal strategy ranks the users according to their channel gain $\beta$. Then the $x$ group is formed with the $K$ first users (users with the highest $\beta$ ). Two approaches are considered in this strategy: the maximum channel path gain per antenna and the mean channel path gain.

1) $\beta_{\max }$ : In this case the metric relies on the selection of the $K$ users with the highest beta to any $m$ base station antenna:

$$
\underset{k \in \chi}{\arg \max } \beta_{k}(\mathrm{~m}) .
$$

2) $\beta_{\text {mean }}$ : This selection algorithm selects the $K$ users with the highest mean channel gain between to the $M$ antennas at the base station.

$$
\underset{k \in \chi}{\arg \max } \sum_{\mathrm{m}}^{M} \frac{\beta_{k}(m)}{M}
$$




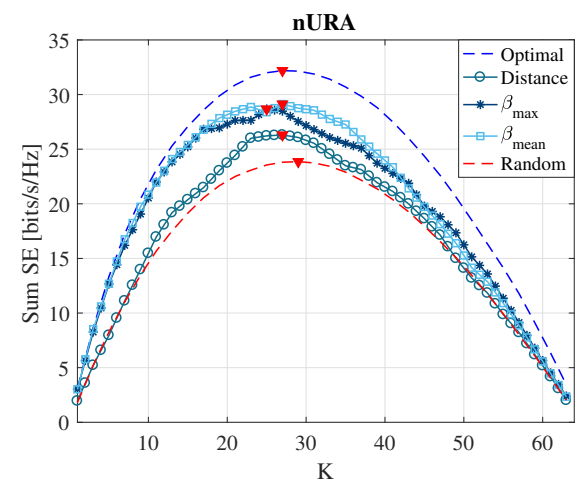

Fig. 2. Sum SE comparison for different users grouping techniques in function of the number of selected users $K$, for the nLoS URA scenario (nURA).

\section{RESULTS}

In this section, the previous user grouping algorithms are applied to the measurement database, where 120 users are located in a relatively small area, for multiple antenna topologies and LoS versus nLoS conditions. On the one hand, the impact of the sum SE is analysed over the multiple antenna configurations and the number of selected users. On the other hand, the spectral efficiency per user is analysed during the multiplexing, saturation and scheduling regime.

\section{A. Sum Spectral Efficiency}

1) $n L o S$ Scenario: In the case of the nLOS scenario with a URA antenna distribution, we see in Fig. 2 that both $\beta$-techniques tend to perform well during the multiplexing regime $(K \leq 10)$. Meanwhile, in the same regime, the method based on distance provides similar behaviour as the random selection method. This can be understood, as in a nLoS scenario, geometric distance is not a good prediction of the channel quality. During the saturation regime, $(10 \leq K \leq 35)$ all presented techniques offer a higher SE than the random selection. However, none of the $\beta$ neither distance techniques are close to the optimal case. Finally, during the scheduling regime $(35 \leq K)$, we can see that none of the proposed methods significantly improve the sum SE in comparison with a random user selection. In this figure, the saturation points are the red triangles for each grouping technique.

2) LoS Scenarios: For the case of the LoS scenarios, the saturation point $(\Lambda)$ is an interesting reference mark to analyse the performance of the different grouping techniques as function of the antenna distribution. The saturation point represents the maximum number of users that can be served to achieve the highest sum SE. Table I shows that the URA case reaches $\Lambda$ with the least number of $K$ selected users, regardless of the user grouping technique. On the other hand, D-ULA supports more users until $\Lambda$ is reached. Furthermore, using the optimal user grouping technique, it is possible to serve almost $1 / 3$ more of the users in comparison with the URA case, due to the reduction of inter-user-interference (IUI), as was described in [7].
TABLE I

NUMBER OF USERS $K$ NEEDED TO ACHIEVE THE SATURATION POINT $(\Lambda)$ IN THE LOS SCENARIOS

\begin{tabular}{|l|c|c|c|}
\hline User Grouping Method & URA & ULA & D-ULA \\
\hline Optimal & 25 & 38 & 43 \\
$\beta_{\max }$ & 25 & 35 & 35 \\
$\beta_{\text {mean }}$ & 25 & 32 & 33 \\
Distance & 17 & 35 & 41 \\
Random & 27 & 33 & 37 \\
\hline
\end{tabular}

The analysis of the sum SE as function of the number of selected users is presented in Fig. 3 for the different LOS scenarios, all saturation points and their sum SE values are presented as the red triangles on each user grouping. Here it is evident that on the saturation point of D-ULA has the highest SE (with the optimal user grouping technique) in comparison with the other antenna topologies.

The same figure also reveals that during the multiplexing period $(K<<\Lambda)$ the sum SE increases steadily with the number of selected users $K$. There is a slight difference, however, between the various antenna distributions. On the one hand, for the URA case, as shown by Fig.3(a), the $\beta$ based user grouping methods improve the SE slightly in comparison with the random selection method. On the other hand, during the same scheduling regime, when considering the ULA and D-ULA antenna distributions, Fig. 3(b) and 3(c) show that different user grouping techniques do not contribute to a difference in the sum SE.

During the saturation regime $(\mathrm{K}= \pm \Lambda)$, none of the heuristics user grouping techniques impact the performance of the sum SE significantly and are close to sum SE values given by a random user selection. In the ULA scenario, only the distance grouping technique has a marginally better performance than a random selection, due to the symmetry of the scenario. Finally, in the scheduling regime $(\mathrm{K}>>\Lambda)$, Fig. 3(a) and 3(b) show that a random user grouping provides a higher SE than the proposed methods. That is not the case for the D-ULA antenna distribution; here, the user grouping based on distance is a better choice than the random selection.

\section{B. Per User Spectral Efficiency}

The Inter-Quartile-Range (IQR) measures the variability of the user SE; therefore, when the inter-quartile-distance becomes more significant, the user fairness is lower. The IQR metric and its values are presented in Fig. 4. The figure compares the different user grouping techniques using different antennas distribution in different regimes, for the nLoS case (nURA) and all the LoS antenna topologies.

During the multiplexing regime $(K=9)$ in Fig. 4(a), it is noticeable that URA provides the highest mean user SE (red line inside each boxplot) regardless of the user grouping technique. Nevertheless, the same antenna geometry shows a large variation of the user SE values. In the case of the DULA, all the grouping techniques show better user fairness, except for the distance user grouping technique. 


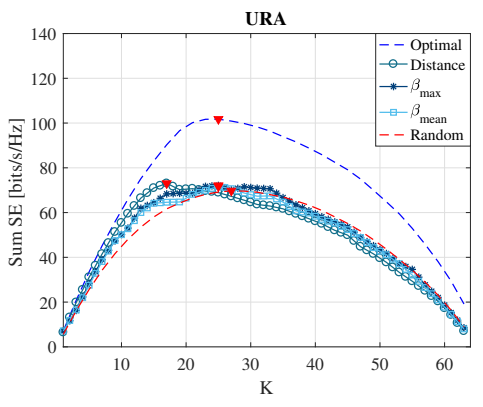

(a) $8 \times 8$ URA antenna.

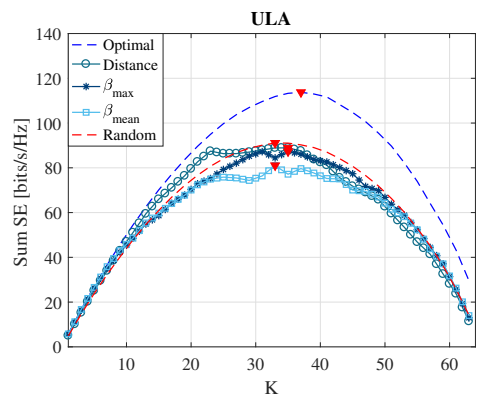

(b) $1 \times 64$ ULA antenna.

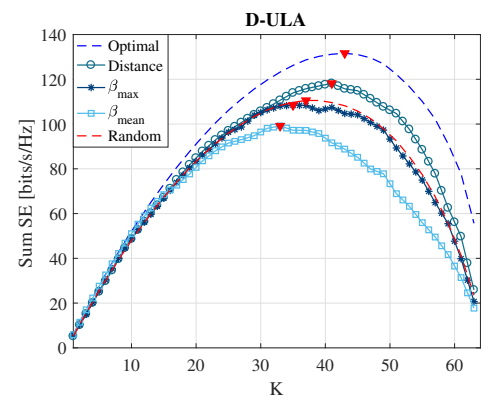

(c) 8 distributed antenna arrays of $1 \times 8$, DULA.

Fig. 3. Sum SE comparison for different users grouping techniques, the number of selected $K$ users, and antenna topology for LoS scenarios.

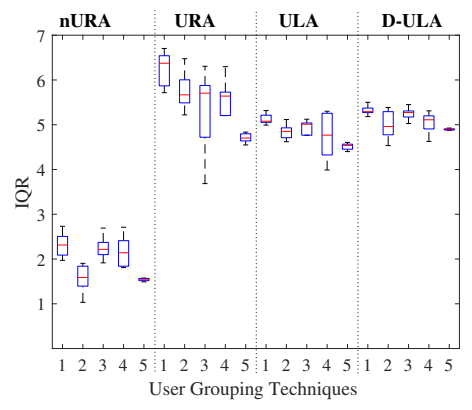

(a) Mutiplexing Regime $K=9$.

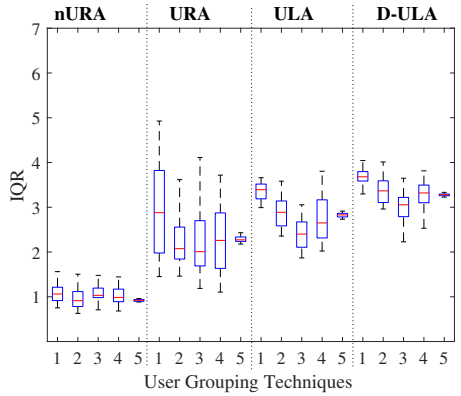

(b) Saturation Regime $K=35$.

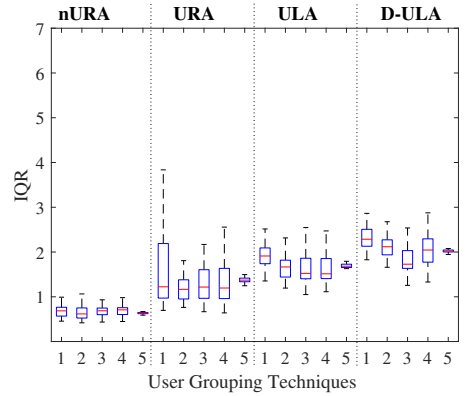

(c) Scheduling Regime $K=55$.

Fig. 4. IQR of user SE for the different antenna distribution, $K$ users and user grouping techniques: 1. Optimal, 2. Distance, 3. $\beta_{\max }, 4$. $\beta_{\min }, 5$. Random.

During the saturation regime $(K=35)$, as shown in Fig. 4(b), the URA case shows the worst user fairness again for all user scheduling techniques (except random grouping). The DULA case shows to have the highest mean user SE for all the user grouping techniques, except the optimal user grouping technique in ULA. The same behaviour is also noticed during the scheduling regime $(K=55)$, as shown in Fig. 4(c).

\section{Conclusions}

In this work, three multiple user grouping techniques were proposed and analysed using a dense channel database obtained from an indoor 64-antenna system, but for different array distributions, and under a different number of served users. On the one hand, the proposed user grouping techniques impact the performance in a nLoS URA scenario but only in the multiplexing and part of the saturation regime. On the other hand, in LoS scenarios, the presented user scheduling techniques do not impact the performance of the system significantly. However, the array geometry and distribution do have a significant influence on the peak sum $\mathrm{SE}$ of the system. For this experiment, the D-ULA is capable of serving $1 / 3$ more users than the URA antenna geometry, confirming that in a distributed antenna array scenario, the IUI impact is reduced. Therefore, a higher sum SE can be achieved, in comparison to the centralised ULA and URA.

\section{ACKNOWLEDGMENT}

This work was funded by the European Union's Horizon 2020 under grant agreement no. 732174 (ORCA project).

\section{REFERENCES}

[1] T. L. Marzetta, "Noncooperative cellular wireless with unlimited numbers of base station antennas," IEEE Transactions on Wireless Communications, vol. 9, pp. 3590-3600, Nov 2010.

[2] W. Boukley Hasan, P. Harris, A. Doufexi, and M. Beach, "Real-time maximum spectral efficiency for massive mimo and its limits," IEEE Access, vol. 6, pp. 46122-46133, 2018.

[3] E. Björnson, J. Hoydis, and L. Sanguinetti, "Massive MIMO networks: Spectral, energy, and hardware efficiency," Foundations and Trends ${ }^{\circledR}$ in Signal Processing, vol. 11, no. 3-4, pp. 154-655, 2017.

[4] E. Castañeda, A. Silva, A. Gameiro, and M. Kountouris, "An overview on resource allocation techniques for multi-user mimo systems," IEEE Communications Surveys Tutorials, vol. 19, no. 1, pp. 239-284, 2017.

[5] E. Björnson, E. G. Larsson, and M. Debbah, "Massive mimo for maximal spectral efficiency: How many users and pilots should be allocated?," IEEE Transactions on Wireless Communications, vol. 15, Feb 2016.

[6] J. Hoydis, S. ten Brink, and M. Debbah, "Massive mimo in the ul/dl of cellular networks: How many antennas do we need?," IEEE Journal on Selected Areas in Communications, vol. 31, February 2013.

[7] C. Chen, Q. Wang, A. Gaber, A. P. Guevara, and S. Pollin, "Experimental study of user selection for dense indoor massive mimo," in IEEE INFOCOM 2019 - IEEE Conference on Computer Communications Workshops (INFOCOM WKSHPS), pp. 602-607, April 2019.

[8] V. Kotzsch, C. Felber, S. Pollin, T. Vermeulen, M. Danneberg, R. Bomfin, W. Liu, I. Moerman, I. Seskar, A. Nahler, F. Paisana, and L. DaSilva, "Orchestration and reconfiguration control architecture, sc2: Definition of showcases."

[9] S. D. Bast, A. P. Guevara, and S. Pollin, "Csi-based positioning in massive mimo systems using convolutional neural networks," 2019. 